\title{
Be Wary Of Hyperglycaemia In The Newborn A Case Of Monogenic Permanent Neonatal Diabetes (PND)
}

M.Sumbwanyambe, R.Oba, L.lbrahim, K. Tharian. ,Diana Princess Of Wales Hospital, Grimsby.

\section{Introduction}

- A small for gestational age term baby boy (Birth weight $2.3 \mathrm{~kg}$ ) was admitted to the neonatal unit with suspected sepsis and for monitoring blood glucose, being an infant of diabetic mother.

- Contrary to expectation, baby had hyperglycemia 6-12 mmol/L

( initially attributed to sepsis)

- Hyperglycemia persisted despite antibiotic treatment and baby remaining well.

- Parents -non-consanguineous /African origin.

- Mother -has type 1 diabetes with quadriplegic cerebral palsy/ wheel chair bound; presented at one month of age with Diabetic ketoacidosis and coma in Malawi when diagnosed to have diabetes

- Mother's diabetes -fairly well controlled on multiple daily insulin injections; No other family history of diabetes

\section{Investigation}

- Genetic testing -Both baby and mother heterozygous for a previously reported Insulin (INS) missense mutation, p.L30P.

- Result confirms a diagnosis of PND due to an INS gene mutation

- Each of the patient's offspring is at 50\% risk of inheriting this mutation and developing PND.

\section{Management}

- On day 9 of life baby was started on insulin determir 0.5 unit subcutaneously.

- On day 20- changed to continuous subcutaneous insulin infusion (CSII).

- Initially on flat basal profile of rapid acting insulin analogue (Humulog/Lispro) at a dose of 0.05 units/hour, requiring a total daily dose(TDD) of 1 unit/day .

Currently on insulin > 1 unit $/ \mathrm{kg} /$ day with TDD of $6.2 \mathrm{units} / \mathrm{day}(30 \%$ basal; $70 \%$ bolus with(formula) feeds.

- Insulin : carbohydrate ratio 1unit : 22 gram

- Insulin sensitivity 1unit : $22 \mathrm{mmol} / \mathrm{I}$

- Target blood glucose : 5-10 mmol//

Baby is developing normally with latest weight $5.95 \mathrm{~kg}$ (50th centile).

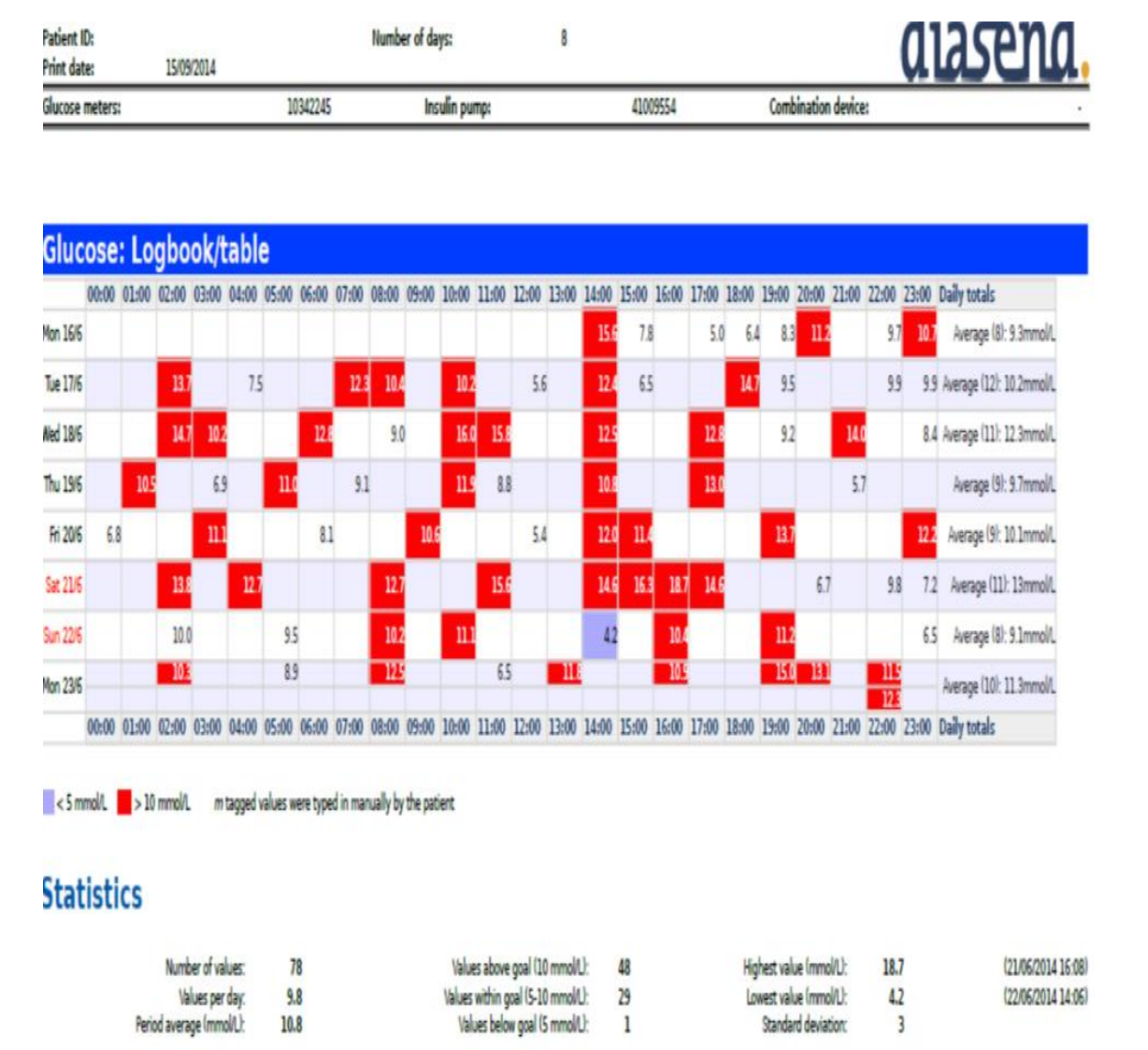

GLYCAEMIC PROFILE AT INITIATION OF CSII

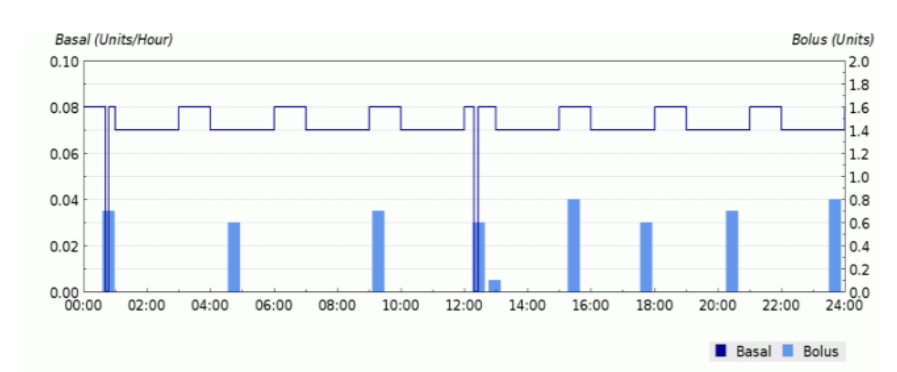

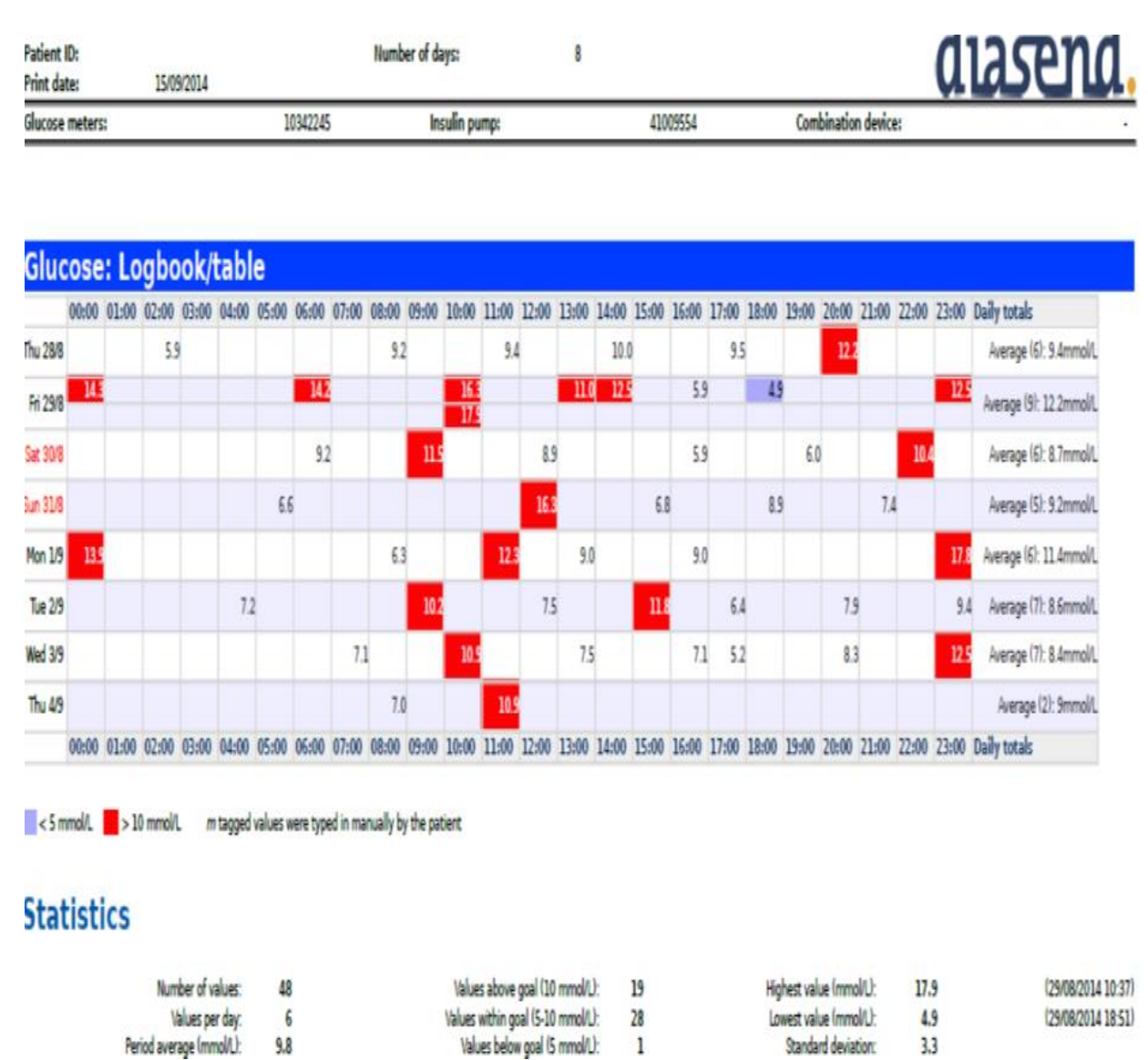

GLYCAEMIC PROFILE 3 MONTHS POST CSII

\section{Discussion}

Neonatal diabetes is a very rare condition with an estimated incidence of 1 in $100,000-500,000$ live births. Fifty percent of these will be permanent and the other $50 \%$ are transient in which case there is remission after a few months ,but may reoccur months or years later.

Neonatal diabetes should not be seen as congenital form of type 1 diabetes, as there is no evidence of insulin or other autoimmune antibodies present in this age group.

GENETIC AETIOLOGIES: Several genetic mutations resulting into permanent neonatal diabetes have been reported. Prevalence of these are as below :

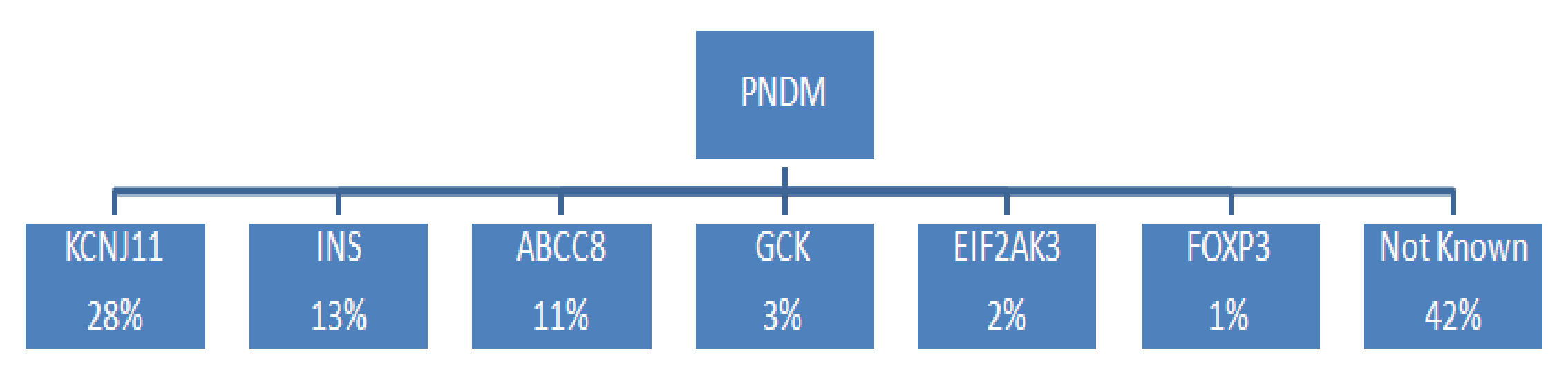

\section{Conclusion}

The management of neonatal diabetes can be quite challenging

It is uncommon with limited knowledge among health professionals.

There is usually associated IUGR as in this case, making subcutaneous cannula for insulin pump a challenge.

$\square$ Sick neonates can be generally metabolically stressed.

$\square$ The need for very small doses of insulin.

Extremely unpredictable blood sugars levels, with frequent feeds.

In our case -young parents with first baby and mother with special needs requiring round the clock care ,made management more challenging for family and team .

Multi agency support services including psychology in place

Suspecting the diagnosis and prompt genetic testing facilitate early diagnosis and appropriate treatment.

REFERENCES:

1. J.Paediatric Child Health (2003) 41, 522-526

2. DIABETES, VOL 57, APRIL 2008

3. www.pnas.org/cgi/doi/10.1073/pnas.07072911104

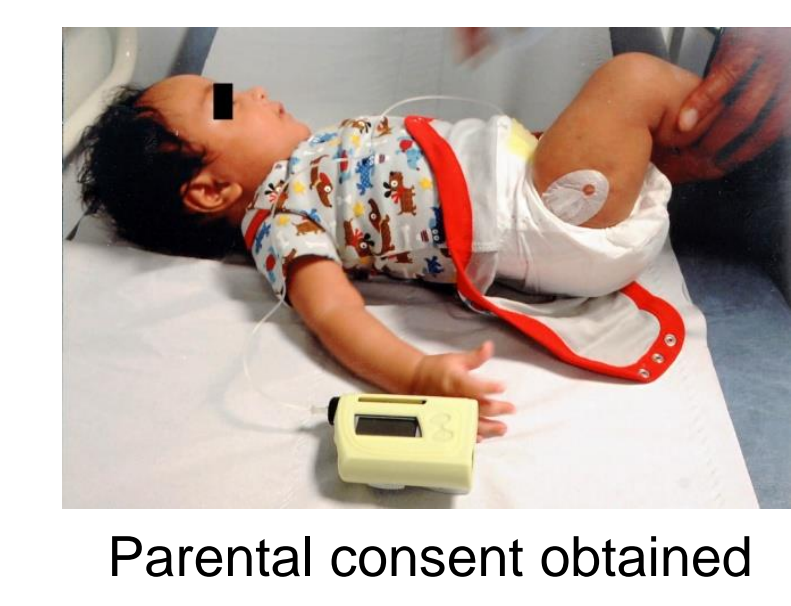

ACKNOWLEDGEMENT: We acknowledge the support of the parents who have given us the consent to report the case. We also thank the Molecular genetics team ,University of Exeter for helping promptly with the genetic testing. Above all we appreciate all the help of the paediatric medical and nursing team who supported the family . 